\title{
Effect of toe exercises and toe grip strength on the treatment of primary metatarsalgia
}

\author{
Kentaro Amaha* ${ }^{*}$, Tatsuya Arimoto and Nobuto Kitamura
}

\begin{abstract}
Background: The relationship of metatarsalgia and toe function is poorly understood. We investigated the efficacy of toe exercises for the treatment of metatarsalgia.

Methods: Forty-one (56 feet) metatarsalgia patients (mean age \pm SD: $63.4 \pm 10.6)$ underwent toe strength measurement. We recorded pre- and post-treatment VAS score, AOFAS score, marble pickup, single-leg standing time (SLST), and compared in two subgroups to evaluate impact of disease duration on treatment outcome.

Results: Post treatment, toe plantarflexion strength improved (all $p<0.01$ ); VAS scores decreased $(p<0.01$ ); AOFAS scores, marble pickup, and SLST improved (all $p<0.01$ ). Patients symptomatic for $>1$ year had significantly lower changes in VAS scores $(p<0.01)$. Multivariate analysis showed patients with longer disease duration, and larger body mass index had significantly lower improvement in VAS scores ( $p=0.029$ and $p=0.036$, respectively). Device consistency assessed by ICC was excellent (0.89-0.97).
\end{abstract}

Conclusion: Toe function and metatarsalgia are improved by toe exercises, suggesting that they are closely related.

Keywords: Toe exercise, Metatarsalgia, Conservative treatment, Toe grip strength, Chronic pain

\section{Introduction}

Muscle mass decreases after approximately age 50 [1]. This age-related loss of muscle mass is termed "sarcopenia" and has been a focus of constant attention because of its association with mortality [2]. Lower ambulatory performance with aging is closely related to sarcopenia $[3,4]$. In the lower extremities, the toes play a crucial role and assist stability during gait and balance tasks [5]. However, elderly patients tend to have decreased toe grip strength (TGS) of approximately $30 \%$, compared to younger patients [6]. Loss of toe-muscle strength leads to impaired balance, thereby increasing the risk of falls [5]. Furthermore, toe grip weakness has been theorized

\footnotetext{
* Correspondence: amaken@luke.ac.jp

Department of Orthopedic Surgery, St Luke's International Hospital, 9-1

Akashicho, Chuo-ku, Tokyo 104-8560, Japan
}

to be associated with a range of forefoot deformities and disorders [7].

Metatarsalgia is one among the commonest conditions causing forefoot pain, characterized by pain in the front part of the foot, under the heads of the metatarsal bones. Metatarsalgia has various causes, including mechanical and iatrogenic factors [8]. Mechanical overload of the weightbearing structure is the fundamental etiology of primary metatarsalgia. Excessive forefoot loading is related to intrinsic abnormalities of metatarsal anatomy and gait mechanics [9]. With regard to gait mechanics, the toes play an important role in maintaining floor contact, which is shared with the metatarsal area during the toe-off phase in the gait cycle [10]. Therefore, the load on the forefoot is affected by the load on the toes. Thus, metatarsalgia seems to be closely related to the toe function.

(c) The Author(s). 2020 Open Access This article is licensed under a Creative Commons Attribution 4.0 International License, which permits use, sharing, adaptation, distribution and reproduction in any medium or format, as long as you give appropriate credit to the original author(s) and the source, provide a link to the Creative Commons licence, and indicate if changes were made. The images or other third party material in this article are included in the article's Creative Commons licence, unless indicated otherwise in a credit line to the material. If material is not included in the article's Creative Commons licence and your intended use is not permitted by statutory regulation or exceeds the permitted use, you will need to obtain permission directly from the copyright holder. To view a copy of this licence, visit http://creativecommons.org/licenses/by/4.0/ The Creative Commons Public Domain Dedication waiver (http://creativecommons.org/publicdomain/zero/1.0/) applies to the data made available in this article, unless otherwise stated in a credit line to the data. 
There have been no reports of the relationship between toe function and primary metatarsalgia. However, toe function is difficult to evaluate. One of the methods to evaluate toe function is to measure the TGS. Despite a few reports on TGS measurement $[6,11]$, as yet, no fully validated study has assessed the absolute value of TGS in a clinical setting [12]. The lack of an evaluation method makes it difficult to understand complicated forefoot disorders. Horiuchi [13] developed a new device that measures TGS: a push-type toe-grip meter that can easily assess toe function.

This study was conducted to investigate TGS in metatarsalgia patients using the abovementioned novel device. Furthermore, we observed the effect of toe exercise on metatarsalgia to assess the relationship between TGS and clinical disease symptoms. We theorized that toe exercise can improve both TGS and clinical symptoms of metatarsalgia.

\section{Methods}

\section{Patients}

This single-center study was approved by the institutional review board (approval no. 17-R069) and conducted in accordance with the tenets of the Declaration of Helsinki. Between April 2012 and December 2015, all patients with metatarsalgia longer than 2 months without remission, regardless of treatment, were included in this study. Metatarsalgia was defined as weightbearing pain and/or tenderness on the plantar side of the lesser metatarsal head. Sixty patients were screened and examined according to the subject selection criteria by a single foot and ankle surgeon (KA) study investigator. As this was a retrospective case series, a statistically powered sample size was not calculated. Simple radiography of the patients' feet was carried out, during weightbearing in the dorsoplantar view. The rate of patients' complications of the hallux valgus, defined as a $20^{\circ}$ or greater angle of the hallux valgus, was examined. Moreover, the forefoot length was assessed in the first metatarsal relative to the second metatarsal. Patients were divided into 3 groups according to this length: in the index minus, the first metatarsal was shorter than the second and the following metatarsals became progressively shorter; in index plus, the first metatarsal was larger than the second; and in index plus-minus, the first and second metatarsals were approximately the same length. Furthermore, all patients underwent MRI and were confirmed not to have rupture of plantar plates or Freiberg's disease. The exclusion criteria were as follows: patients with conditions such as rigid forefoot deformities (e.g., hammer toes, claw toes), patients with infection, crystal arthritis, previous foot surgery, major trauma, rheumatic disease, and neurological disease (e.g., cerebral infarction, parkinsonism, radiculopathy, and Morton's neuroma). After implementing the exclusion criteria, we included 56 feet (41 patients) older than age 20 who were not allowed to take medications or receive any insole treatment during the study period. All patients provided written informed consent.

\section{Toe exercise}

Patients received an 8-week toe functional exercise program conducted under a physiotherapist's guidance. We administered simple exercises such that elderly individuals could understand them. The patients sat in an upright position, placed a towel on the floor, and placed one foot on it, one shoulder-width apart. Then, they used their toes to scrunch up the towel, making sure to keep the rest of their foot in contact with the ground. Three sets of 15 scrunches were performed on each foot. In addition to the towel exercise, curling and spreading out of all toes was done. Patients were instructed to perform the exercises for 10 min 2 times a day for 2 months.

\section{Toe-grip strength meter}

TGS was measured using the push-type toe-grip strength meter (Fig. 1) [13]. Using the strain gauge installed in the cantilever, the device measures the strength of the toes pressing the floor. For the measurement, a foot was placed on the positioning bar of the device and strapped tightly to restrict upward motion during measurement. The measurement was started after the confirmation of zero on the device's screen. The second foot could be measured by turning the measurement board upside down. We measured all the TGS values before and after the exercise program in both upright and sitting positions. In the upright position, patients stood upright on the measurement board facing forward. Special care was taken not to have the patient's head down, causing extra weight to be placed on the toes. During measurement in the seated position, patients sat on a chair adjusted to their height with their

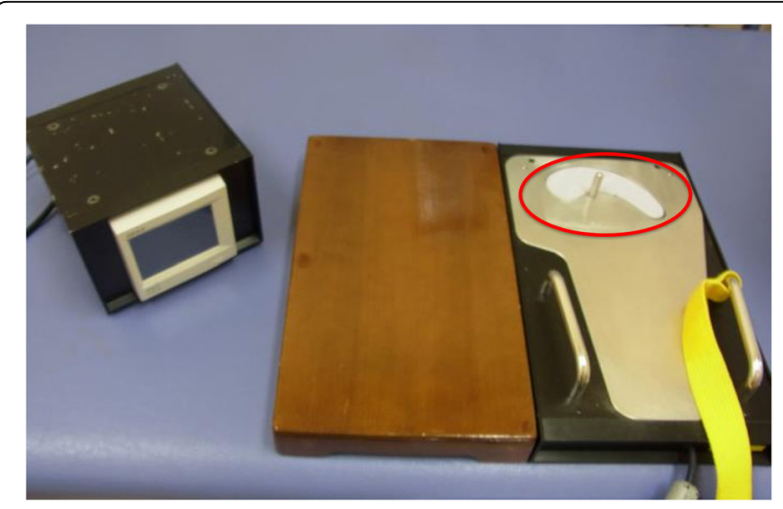

Fig. 1 Push-type toe-grip strength meter. Toes press the floor, facing the strain gauge installed in the cantilever (red circle) 
knee and hip joints precisely at $90^{\circ}$. Then, the patients gripped their toes using maximal force in both positions. The highest value (Newton: $\mathrm{N}$ ) of force with which toes were gripped was automatically recorded as the maximal peak force on the device's screen (Fig. 2). We undertook the measurement twice on the same day. The average of the two measured values was recorded.

\section{Assessment}

Patients were also assessed by using the visual analog scale (VAS) for pain, the American Orthopedic Foot and Ankle Society (AOFAS) hallux metatarsophalangeal-interphalangeal scale (scale of 100 points), the number of marbles that the patient could pick up using their toes in $10 \mathrm{~s}$ while seated (picking up the marbles [PUM]) and observing the number of seconds the patient was able to stand on a single leg for up to $60 \mathrm{~s}$ (single-leg standing time [SLST]). The VAS, consisting of horizontal lines of a 100-mm length, was self-recorded. For pain intensity, the scale is anchored by "no pain" (scale of 0 ) and "worst imaginable pain" (scale of 100). To investigate whether the duration of disease affects the outcome of treatment, patients were divided into two groups based on disease durations of more than 1 year or less, and the average difference in the degree of improvement between the pre- and post-treatment measures was examined. Multivariate analysis was conducted to investigate which background factor correlated with the degree of improvement in the TGS in the upright and sitting positions, degree of improvement in AOFAS score, and improvements in VAS scores. The reliability of the push-type toe-grip strength meter was assessed with the Bland-Altman plot using the intra-rater correlation coefficient (ICC). During the measurement of TGS, the inter-rater reliability was not examined because the value is only read off of the screen of the device.

\section{Statistical analysis}

To compare the pre-exercise (pre-ex) and post-exercise (post-ex) values in relation to the parameters evaluated, the paired $t$ test for independent samples was used. The average difference in the degree of improvement between the pre- and post-treatment measures was also evaluated by the paired $t$ test. A multivariate generalized linear model, using normal distribution, was used to investigate the relationship between the background factors, including age, sex, body mass index (BMI), duration of disease, affected side, forefoot morphology, and outcomes, including improvement of TGS in upright and seated positions, VAS scores, and AOFAS scores. In the affected-side analysis, the right and left feet were evaluated as a comparison based on bilateral feet measurements. In the forefoot morphology, plus and plus-minus feet were evaluated as a comparison based on minus feet. To determine the interrater reliability of the push-type toe-grip strength meter, the Bland-Altman plot was constructed to assess the agreement. Intra-class correlation coefficients for agreement were calculated. The data were analyzed by SPSS statistical software (version 7, Chicago, Illinois). The level of significance was set at $p<0.05$.

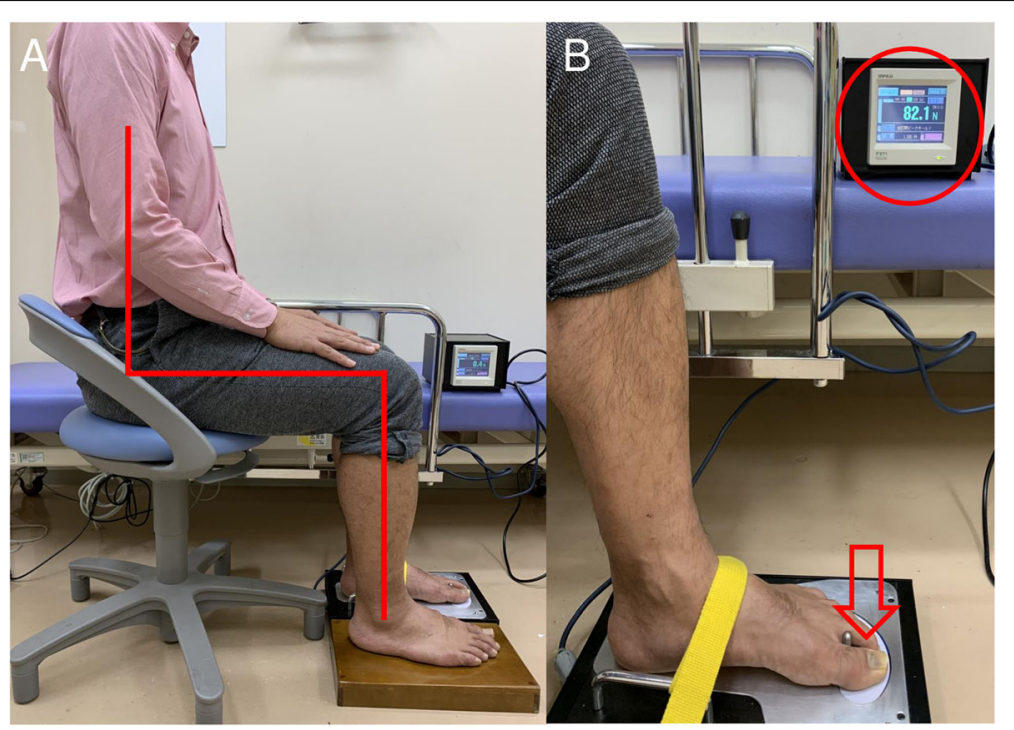

Fig. 2 Measurement of TGS. a Measurement in the seated position, foot is placed on the positioning bar and strapped tightly to the device. The patients sat on a chair adjusted to their height with their knee and hip joints precisely at $90^{\circ}$. Then, the floor is pressed as firmly as possible with the foot. $\mathbf{b}$ The greatest value (Newton: N) while the toes are gripping (red arrow) is automatically recorded as the maximal peak force on the black box screen (red circle) 


\section{Results}

Basic characteristics of the current study participants are shown in Table 1. In total, 56 feet with metatarsalgia, 32 on the right side and 24 on the left side, were included. Symptoms involved both feet in 14 patients. The average duration of metatarsalgia was $21.2 \pm 33.2$ (range 2-120) months. In the evaluation of forefoot morphology, index minus was present in two thirds of patients, and index plus was only present in 1 case. A total of 20 patients, $30.3 \%$ of whom had a hallux valgus angle of $20^{\circ}$ or more on foot X-ray (Table 1).

In the unilateral cohort, in the sitting position, the grip strength of the toe of the affected foot was significantly weaker at $23 \pm 17.2 \mathrm{~N}$ compared to $28.8 \pm 18 \mathrm{~N}$ on the unaffected side $(p<0.01)$. In the upright position, the strength of the affected foot was $51.8 \pm 37.9$, which was weaker than the strength of the unaffected foot at $57.8 \pm$ $31.3 \mathrm{~N}$, but this difference was not significant. After the 8-week exercise program, all variables, including toe plantar-flexion strength, significantly increased in both standing and seated positions. Table 2 shows the change in values. The mean VAS score significantly decreased from 5.2 to $2.5(p=0.00)$. The AOFAS score significantly improved from 67.2 to $77.1(p=0.00)$ and PUM also significantly improved from 7.7 to $9.5(p=0.00)$.

After dividing patients into two groups according to disease duration, there were 20 patients in the more than 1-year group and 36 in the less than 1-year group. With the available numbers, no significant difference in TGS or AOFAS scores could be detected pre- and postexercise in neither upright nor sitting positions. Only VAS scores were significantly different between the two groups. Patients with symptoms persisting for more than 1 year showed a significantly lower change in VAS scores (Table 3). The multivariate generalized linear model showed that the plus-minus foot had significant improvement in AOFAS scores compared to that of the minus foot between the upright and sitting positions. Regarding VAS scores, improvement was worse in

Table 1 Basic characteristics

\begin{tabular}{ll}
\hline Variables & \\
\hline Age (years) & $63.4 \pm 10.6$ \\
Gender, male/female & $7 / 34$ \\
Height (cm) & $156.6 \pm 7.7$ \\
Weight (kg) & $52.8 \pm 7.3$ \\
BMl (kg/m²) & $21.5 \pm 2.5$ \\
Duration of metatarsalgia (months) & $21.2 \pm 33.2$ \\
Affected side, left/right/bilateral & $10 / 18 / 14$ \\
Hallux valgus (\%) & $17 / 56(30.3 \%)$ \\
Morphology, minus/plus-minus/plus & $38 / 17 / 1$ \\
\hline
\end{tabular}

Values are provided as mean \pm standard deviation Abbreviations: $B M I$ body mass index
Table 2 Pre- and post-exercise values

\begin{tabular}{llll}
\hline & Pre-ex & Post-ex & $\boldsymbol{P}$ \\
\hline Upright TGS (N) & $54.4 \pm 36.1$ & $67.4 \pm 37.9$ & 0.01 \\
Sitting TGS (N) & $24.7 \pm 16.8$ & $32.1 \pm 17.9$ & $<0.01$ \\
AOFAS score & $66.7 \pm 16$ & $79.9 \pm 12.5$ & $<0.01$ \\
VAS & $4.4 \pm 1.6$ & $1.8 \pm 1.4$ & $<0.01$ \\
Marble pickup (number) & $5.9 \pm 2.9$ & $8.6 \pm 2.3$ & $<0.01$ \\
SLST (sec) & $39.4 \pm 23.2$ & $49.3 \pm 19$ & $<0.01$
\end{tabular}

Values are provided as the mean \pm standard deviation

Abbreviations: TGS toe-grip strength, ex exercise, AOFAS American Orthopedic

Foot and Ankle Society, VAS visual analog scale, SLST single-leg standing time

patients with a long history of disease and high BMI (Table 4). The results from the Bland-Altman plots indicated that there was excellent agreement among the positions, both on the affected side and the unaffected side (0.89-0.97). No adverse events were noted.

\section{Discussion}

The unique findings of this study provide objective evidence confirming the effects of toe exercise on metatarsalgia. The use of insoles was the standard treatment for metatarsalgia, and its effect was reported to improve VAS by an average of 1 point [14]. In this study, the average improvement was 2.7 points, and it resulted in less pain than do insoles. Previous reports have evaluated TGS between the sitting and upright positions [15]. They found that there was no difference in the TGS between the upright and sitting positions. Contrary to the previous report, TGS in the sitting position was significantly lower than that in the upright position. The push-type toe-grip strength meter in the current study measures the toes' pressing force applied to the ground, whereas the pull-type meter used in the previous study measures the toes' pulling force in the proximal direction, similar to the hand-grip dynamometer. As the shape of the toes is different for each person, it is questionable whether the pulling bar of the pull-type device fits all foot shapes. Furthermore, we placed emphasis on the force that presses or steps on the ground as a

Table 3 Mean difference between pre- and post-operative measures in two groups of differing disease duration

\begin{tabular}{llll}
\hline Values & Over $\mathbf{1}$ year $(\boldsymbol{n}=\mathbf{2 0})$ & Less than $\mathbf{1}$ year $(\boldsymbol{n}=\mathbf{3 6})$ & $\boldsymbol{p}$ \\
\hline Upright TGS & $15.9 \pm 26.8$ & $11.3 \pm 30.3$ & 0.56 \\
Sitting TGS & $9.2 \pm 8.6$ & $6.3 \pm 15.2$ & 0.35 \\
VAS & $1.5 \pm 1.19$ & $3 \pm 1.5$ & $<0.01$ \\
AOFAS score & $11.7 \pm 10.9$ & $14.1 \pm 10.6$ & 0.42 \\
\hline
\end{tabular}

Values are provided as the mean \pm standard deviation

Abbreviations: TGS toe-grip strength, ex exercise, AOFAS American Orthopedic Foot and Ankle Society, VAS visual analog scale 


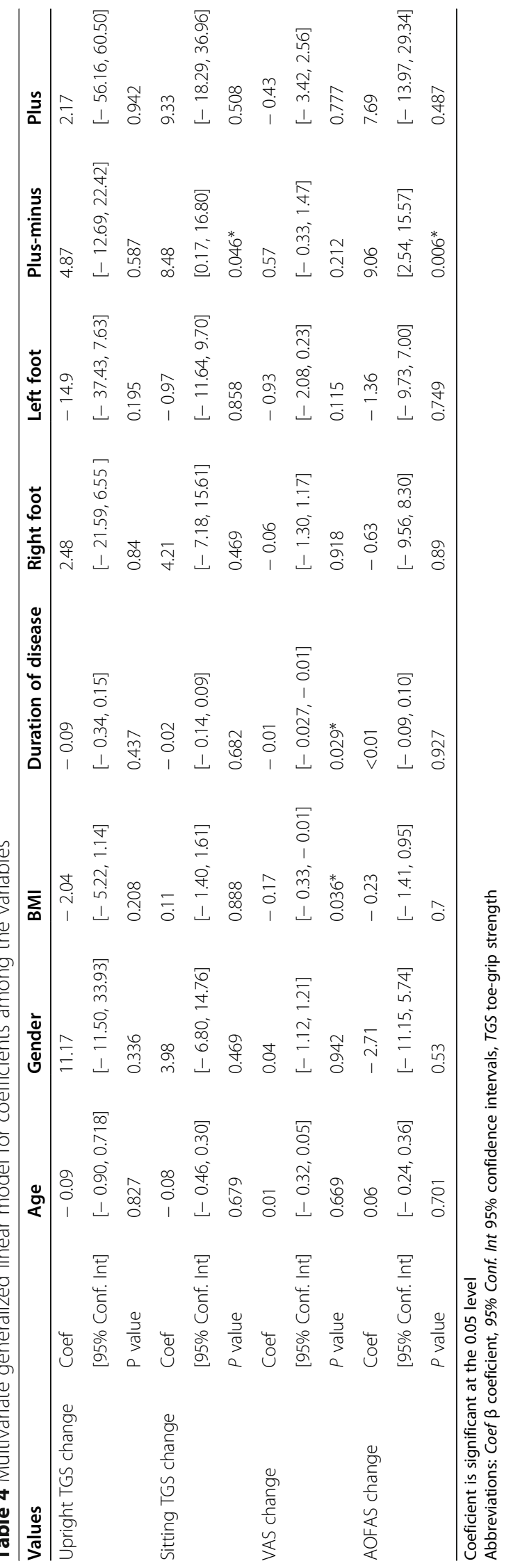


function of the toes. In that sense, we believe that the push-type device reflects the function of the toes more clearly.

The current study also demonstrated that the affected foot has significantly weaker TGS in the sitting position compared to that in the unaffected foot, but no significant difference was observed in the upright position. It is unclear why the difference in the upright case is not manifested; it may possibly be due to some compensatory function working in the upright position to maintain the TGS.

Primary metatarsalgia is considered an abnormality that is related to the anatomy of the metatarsal bones as well as to the relationship between metatarsal bones and the rest of the foot, which leads to overload [9]. Metatarsal bone-length discrepancy has received the most attention thus far [16]. The present study showed that patients with index-minus accounted for most of the participants, suggesting that the second metatarsal was relatively long, and the metatarsal length seemed to be involved in the onset of metatarsalgia. However, as the length of the metatarsal bone cannot be changed except for surgery, another viewpoint is required for conservative treatment.

During walking, the load increases to about 2 times that of body weight before toe-off at the MTP joints [17] due to the combined effect of forward-falling and ground reaction force loads applied to the forefoot. When the toes lose the ability to functionally push off the ground, it places an increased load on the metatarsal area. This repetitive overloading in the metatarsal area causes metatarsalgia.

We observed an improvement in PUM, indicating that exercise not only improves strength but also enhances toe function. Therefore, metatarsalgia may be relieved as a consequence of an improvement in toe function.

Patients with long durations of metatarsalgia (more than 1 year) did not have improved VAS scores to a great extent in this study. The multivariate generalized linear model showed that the foot with plus-minus morphology had a significant improvement in AOFAS scores compared to that in the foot with minus morphology in the upright and sitting positions. Regarding VAS scores, improvement was worse in patients with a long history of disease and high BMI. In terms of the long duration of metatarsalgia, chronic pain is generally considered difficult to cure. Although there is an opinion that it is difficult to create a temporal boundary in terms of the difference between acute and chronic pain, generally, pain lasting 12 weeks or more is regarded as chronic pain [18]. The cause of pain in metatarsalgia is unclear, but it is presumed that the acute phase involves nociceptive pain. The improvement in pain was worse in chronic cases, suggesting that mixed pain, involving nociceptive, nociplastic, and neuropathic pain, is involved in chronic disease [19]. For patients with hallux valgus, waiting for elective surgery has been associated with less improvement in physical function outcomes following surgery [20], which supports our findings. Our results suggest that a different treatment strategy may be needed for chronic pain in metatarsalgia lasting more than 1 year.

We found that an increased BMI positively correlated with worse improvement in VAS scores. Moreover, previous reports showed a high BMI was positively correlated with elevated opioid consumption rates [21]. However, this result may stem from doctors prescribing a high number of opioids because of the patient being obese. The relationship between obesity and pain is still unclear.

The strength of this study is in the assessment of the toes through the use of reproducible absolute values of TGS, showing that weak toes tend to promote metatarsalgia. This device measures TGS by measuring the strength used to press the toes into the floor. The force of toe plantar-flexion is the strength of sustaining one's weight, shared with the ball area in the toe-off phase. Therefore, this movement directly reflects the power of the toe-off phase by pressing off the ground. This pushtype toe-grip strength meter is a useful device to detect toe weakness in the clinical setting.

The current study has certain limitations. First, the participants were recruited from a single institution; hence, the results may not be generalizable. Second, we have no objective proof of overloading in the metatarsal area in people with weak toe grip. Proper walking not only needs a certain level of plantar-flexion strength but also the ability to move the toes in order to work effectively. The ability of toes to control the loading warrants further investigation. Third, pain may deteriorate due to the natural course of metatarsalgia progression. Since we did not include a control group, further investigations in the form of randomized controlled studies are required to investigate the effectiveness of toe exercises in metatarsalgia.

\section{Conclusion}

We investigated TGS in metatarsalgia patients by using a new device. To our knowledge, this is the first observational study to determine the relationship between TGS and clinical symptoms. Our results provide objective evidence confirming the effect of toe exercises. Patients with metatarsalgia tend to have a weaker TGS. Regaining toe strength could ease the pain caused by metatarsalgia; however, patients with minus morphology feet are likely to develop metatarsalgia, resulting in difficulty in healing, and patients with chronic metatarsalgia for more than 1 year show a significantly lower improvement in VAS scores. The push-type toe-grip strength meter is highly reproducible and a clinically useful device. 


\section{Acknowledgements}

We are grateful to Daiki Kobayashi, Sachiko Ohde of the St. Luke's Graduate School of Public Health for their kind assistance.

\section{Authors' contributions}

Kentaro Amaha designed the study and wrote the manuscript. Tatsuya Arimoto analyzed and interpreted the patient data. Nobuto Kitamura approved the final version of the manuscript. The authors read and approved the final manuscript.

\section{Funding}

No organization sponsored the research.

\section{Availability of data and materials}

All the data will be available upon motivated request to the corresponding author of the present paper.

\section{Ethics approval and consent to participate}

This study was approved by the institutional review board (approval no. 17R069) of the St. Luke's International Hospital.

Informed consent was obtained from all the participants.

\section{Consent for publication}

Written informed consent was obtained from each patient to authorize the publication of their data.

\section{Competing interests}

The authors declare that they have no competing interests.

Received: 10 July 2020 Accepted: 24 November 2020

Published online: 02 December 2020

\section{References}

1. Janssen I. The epidemiology of sarcopenia. Clin. Geriatr. Med. 2011;27: 355-63.

2. Roubenoff R. Sarcopenia: effects on body composition and function. J. Gerontol. A. Biol. Sci. Med. Sci. 2003:58:1012-27.

3. Hida T, Harada A. Fall risk and fracture. Diagnosing sarcopenia and sarcopenic leg to prevent fall and fracture: its difficulty and pit falls. Clin. Calcium. 2013;23:707-12.

4. Hida T, Shimokata H, Sakai Y, Ito S, Matsui Y, Takemura M, et al. Sarcopenia and sarcopenic leg as potential risk factors for acute osteoporotic vertebra fracture among older women. Eur Spine J. 2015;25:3424-31.

5. Menz HB, Morris ME, Lord SR. Foot and ankle characteristics associated with impaired balance and functional ability in older people. J. Gerontol. A. Biol. Sci. Med. Sci. 2005;60:1546-52.

6. Endo M, Ashton-Miller JA, Alexander NB. Effects of age and gender on toe flexor muscle strength. J. Gerontol. A. Biol. Sci. Med. Sci. 2002:57:M392-7.

7. Menz HB, Morris ME, Lord SR. Foot and ankle risk factors for falls in older people: a prospective study. J. Gerontol. A. Biol. Sci. Med. Sci. 2006;61:866-70

8. Espinosa N, Brodsky JW, Maceira E. Metatarsalgia. J. Am. Acad. Orthop. Surg. 2010;18:474-85

9. Espinosa N, Maceira E, Myerson MS. Current concept review: metatarsalgia. Foot. Ankle. Int 2008:29:871-9.

10. Mann RA, Hagy JL. The function of the toes in walking, jogging and running. Clin. Orthop. Relat. Res. 1979;142:24-9.

11. Soma M, Murata S, Kai Y, Nakae H, Satou Y. An Examination of limb position for measuring toe-grip strength. J. Phys. Ther. Sci. 2014;26:1955-7.

12. Soysa A, Hiller C, Refshauge K, Burns J. Importance and challenges of measuring intrinsic foot muscle strength. J. Foot. Ankle. Res. 2012;5:29.

13. Horiuchi K, Handa S, Aoki K. A design and evaluation of a push type toes flexion strength meter. Jpn J Wellbeing Sci Assist Technol. 2008:8:15-22.

14. The effect of metatarsal padding on pain and functional ability in metatarsalgia. Scand J Surg. 2017;106(4):332-7. https://doi.org/10.1177/ 1457496916683090 Epub 2017 Mar 1.

15. Handa S, HKK, Aoki. A study on the measurement of toes grasping strength and effect of standing postural control, Jpn. J. Ergon. 2004;40:139-47 (in Japanese)

16. Mann RA, Chou LB. Surgical management for intractable metatarsalgia. Foot Ankle Int. 1995;16:322-7.
17. Al-Munajjed AA, Bischoff JE, Dharia MA, Telfer S, Woodburn J, Carbes S. Metatarsal loading during gait-A musculoskeletal analysis. J. Biomech. Eng. 2016;138:4032413

18. Johnson Ml. The landscape of chronic pain: broader perspectives. Medicina. 2019;55(5):182.

19. Freynhagen $\mathrm{R}$, Parada HA, Calderon-Ospina CA, Chen J, Rakhmawati Emril D, Fernández-Villacorta FJ, et al. Current understanding of the mixed pain concept: a brief narrative review. Curr. Med. Res. Opin. 2019;35:1011-8.

20. Sutherland JM, Wing K, Younger A, Penner M, Veljkovic A, Liu G, et al. Relationship of duration of wait for surgery and postoperative patientreported outcomes for hallux valgus surgery. Foot Ankle Int. 2019:40:259-67.

21. Kvarda P, Hagemeijer NC, Waryasz G, Guss D, DiGiovanni CW, Johnson AH. Opioid consumption rate following foot and ankle surgery. Foot Ankle Int 2019;40:905-13.

\section{Publisher's Note}

Springer Nature remains neutral with regard to jurisdictional claims in published maps and institutional affiliations.
Ready to submit your research? Choose BMC and benefit from:

- fast, convenient online submission

- thorough peer review by experienced researchers in your field

- rapid publication on acceptance

- support for research data, including large and complex data types

- gold Open Access which fosters wider collaboration and increased citations

- maximum visibility for your research: over $100 \mathrm{M}$ website views per year

At BMC, research is always in progress.

Learn more biomedcentral.com/submissions 